\title{
Risk assessment model constructed by differentially expressed IncRNAs for the prognosis of glioma
}

\author{
CHENGGONG HU, YONGFANG ZHOU, CHANG LIU and YAN KANG \\ Department of Critical Care Medicine, West China Hospital of Sichuan University, Chengdu, Sichuan 610041, P.R. China
}

Received January 14, 2018; Accepted August 1, 2018

DOI: $10.3892 /$ or.2018.6639

\begin{abstract}
A risk assessment model was constructed using differentially expressed long non-coding (lnc)RNAs for the prognosis of glioma. Transcriptome sequencing of the lncRNAs and mRNAs from glioma samples were obtained from the TCGA database. The samples were divided into bad and good prognosis groups based on survival time, then differently expressed lncRNAs between these two groups were screened using DEseq and edgeR packages. Multivariate Cox regression analysis was performed to establish a risk assessment system according to the weighted regression coefficient of lncRNA expression. Survival analysis and receiver operating characteristic curve were conducted for the risk assessment model. Furthermore, the co-expression network of the screened lncRNAs was constructed, followed by the functional enrichment analysis for associated genes. A total of 117 lncRNAs were screened using edgeR and DEseq packages. Among all differently expressed lncRNAs, five lncRNAs (RP3-503A6, LINC00940, RP11-453M23, AC009411 and CDRT7) were identified to establish the risk assessment model. The risk assessment model demonstrated a good prognostic function with high area under the curve values in the training, validation and entire sets. The risk score was certified as an independent prognostic factor for gliomas. Multiple genes were screened to be co-expressed with these five lncRNAs. Functional enrichment analysis demonstrated that they were involved in cytoskeleton, adhesion and Janus kinase/signal transducer and activator of transcription signaling pathway-associated processes. The present study established a risk assessment model integrating five significantly different expressed lncRNAs, which may help to assess the prognosis of patients with glioma with increased accuracy.
\end{abstract}

Correspondence to: Dr Yan Kang, Department of Critical Care Medicine, West China Hospital of Sichuan University, 37 Guo Xue Xiang, Chengdu, Sichuan 610041, P.R. China

E-mail: huchenggong2017@163.com

Key words: glioma, risk assessment model, prognosis

\section{Introduction}

Glioma is one of the most common primary tumors in brain and it accounts for the majority of central nervous system oncology cases in adults globally in 2016 (1). In general, glioma infiltrates into the brain and affects the cerebral hemispheres of patients (1). Though aggressive therapies including surgery, chemotherapy, and radiotherapy have been developed and applied widely, glioma remains associated with high recurrence rates and there are no efficient treatment options (2). The prognosis of glioma patients remains poor (3). Therefore, the development of novel treatments, the identification of new prognostic biomarkers and a clearer understanding of the molecular mechanisms underlying disease progression are essential and urgently required.

Long non-coding RNAs (lncRNAs) account for $80 \%$ of non-coding RNAs and are defined as the endogenous cellular RNAs that are $>200$ nucleotides in length $(4,5)$. With the development of biological computing research tools including lncRNAdb, ChIPBase, LNCipeida and lncRNAtor, the number of lncRNAs being identified is rapidly increasing. In total, >8,000 lncRNA genes have been identified within 4 years and the number of human IncRNAs is estimated to be between 10,000 and 20,000 (6,7). A previous study revealed that lncRNAs are associated with multiple critical cellular functions, including transcriptional, posttranscriptional and epigenetic functions (8). LncRNAs have attracted considerable attention in cancer, since they may be involved in oncogenic and tumor suppressive pathways (6). In the last decade, accumulating evidence demonstrated that the expression levels of lncRNAs were correlated with the development and progression of several types of cancer, through affecting the biological processes including growth, proliferation, metastasis and invasion $(9,10)$. In addition, the functional roles that they may serve in tumor prognosis have been also investigated (11).

The important roles of lncRNAs in gliomas have been revealed in multiple studies. It was reported that lncRNAs may regulate cellular proliferation, apoptosis and migration in glioma $(3,12,13)$. In addition, aberrant expression of lncRNAs has been linked with differential treatment responses in patients with glioma and lncRNAs may be promising as diagnostic and prognostic biomarkers in glioma (14). Ma et al (15) reported that lncRNA metastasis-associated lung adenocarcinoma transcript 1 (MALAT1) may regulate the progression of glioma, thus serving as a potential prognostic biomarker. Using univariate Cox 
regression analysis, the overexpression of lncRNA AB073614 was identified as a poor prognostic biomarker in glioma (16). Despite these findings, our understanding of the prognostic role of lncRNAs in glioma remains unclear.

In the present study, the lncRNA sequencing (lncRNA-seq) data and mRNA-seq expression profiles of glioma samples were downloaded from the TCGA database and the differently expressed lncRNAs were screened. Furthermore, multivariate Cox regression analysis was used to establish a risk assessment system based on the weighted regression coefficient of lncRNA expression. Subsequently, survival analysis for the risk assessment model was conducted in training, validation and entire sets. Finally, the co-expression networks of lncRNAs in the risk assessment model were constructed and the function enrichment was performed for the genes associated with these lncRNAs.

\section{Materials and methods}

Data and data processing. The lncRNA-seq data and mRNA-seq expression profiles of glioma samples were downloaded from the TCGA database (gdc-portal.nci.nih. gov). Glioblastoma samples were used. A total of 173 glioma samples were obtained. Following matching the barcodes of lncRNA-seq data and mRNA-seq expression profiles, 154 glioma samples remained. Finally, 140 samples were selected for analysis following removal of the samples without survival information and overall survival time $<6$ months. The lncRNAs were annotated using human genome annotation GTF format in the GENCODE database (www.gencodegenes. org) (17). These 140 samples were randomly divided into two groups as the ratio of 1:1, namely training and validation sets.

Screening differently expressed lncRNAs. The 70 glioma tissue samples in the training group were divided into bad prognosis (patients succumbed within 6 months) and good prognosis (patients with a survival time $>12$ months) groups. Subsequently, the differentially expressed lncRNAs between these two groups were screened using DEseq 1.16.0 (www.bioconductor. org/packages/release/bioc/html/DESeq.html) (18) and edgeR 3.8.5 (www.bioconductor.org/packages/release/bioc/html/edgeR. html) (19) package in R 3.1.0 (www.r-project.org) with the threshold of false discovery rate (FDR) $<0.05$ and $\log _{2}$ fold changel $>0.263$. Then, the differently expressed lncRNAs were screened for prognostic factors (threshold, $\mathrm{P}<0.05$ ) using the log-rank test and univariate Cox regression analysis in $\mathrm{R}$ 3.1.0.

Establishment of a risk assessment model. Based on the previously obtained differentially expressed lncRNAs, multivariate Cox regression analysis was performed to establish a risk assessment system according to the regression coefficient weighted lncRNA expression. The risk score for each patient was based on a linear combination of mRNA expression values after weighting regression coefficients. The risk assessment for each patient was scored according to the following equation: Risk score $=\beta_{\text {IncRNA1 }} \times \operatorname{expr}_{\text {lncRNA1 }}+\beta_{\text {IncRNA2 }} \mathrm{x}$ $\operatorname{expr}_{\text {lncRNA2 }}+\cdots+\beta_{\text {IncRNAn }} \times \operatorname{expr}_{\text {lncRNAn }}$, where expr means the expression level of lncRNA. The $\beta$ value obtained in the training set was also used to assess the risk of patients in the validation set.
Survival analysis for risk assessment model. The risk score of samples in validation set was calculated according to the risk assessment system. Subsequently, the samples were divided into high and low-risk types based on the median risk score. Finally, Cox regression was performed for correlation analysis in combination with corresponding clinical data of samples. Kaplan-Meier survival analysis was performed to compare the overall survival (OS) rates of patients with high and low-risk scores. Receiver Operating Characteristic (ROC) curve was used to evaluate the classification efficiency of the obtained risk assessment model. Furthermore, the distribution of signature lncRNAs in training validation and entire sets were analyzed and displayed. In addition, the Kaplan-Meier survival analysis and ROC curve were also performed for each of these lncRNAs in the risk assessment model.

Correlation between risk assessment model and clinical features. The risk scores of samples in the validation set were calculated according to the risk assessment system. Subsequently, the samples were divided into high and low-risk types based on the median risk score. The correlations between prognosis and the clinical features, including risk score, age, sex, chemotherapy and pharmaceutical-therapy, were evaluated using univariate and multivariate Cox regression analyses.

Subsequently, hierarchical analysis was performed for the screened clinical features, which were significantly correlated with prognosis risk. The association between the risk groups and survival prognosis was analyzed under the same clinical condition.

Construction of co-expression network. The mRNA expression data matching the lncRNAs was identified based on the sample number. The co-expression network of lncRNA and mRNA was constructed using MEM software $(20,21)$ with the screening threshold of $\mathrm{P}<0.05$. Then, the interaction between the mRNAs in the co-expression network was identified using the STRING (string-db.org) database (22). The interaction pairs with interaction score above 0.4 were selected. Cystoscope 3.4 (www.cytoscape.org) was used to visualize the networks (23).

Functional enrichment analysis. To identify the signaling pathways and biological processes that may be associated with the prognosis of gliomas, Gene Ontology (GO) analysis and Kyoto Encyclopedia of Genes and Genomes (KEGG) enrichment analysis were performed for the mRNAs, which were co-expressed with the lncRNAs in risk assessment model, by Database for Annotation, Visualization and Integrated Discovery (david.ncifcrf.gov) (24) with $\mathrm{P}<0.05$.

Statistical analysis. In the present study, the data were presented as the mean \pm standard deviation. An unpaired t-test was used to compare the difference between two groups using $\mathrm{R}$ 3.1.0. $\mathrm{P}<0.05$ was considered to indicate a statistically significant difference.

\section{Results}

Differentially expressed lncRNAs. The clinical information for the training, validation and complete sets is provided in Table I. For the training set, a total of 171 differentially 
Table I. Clinical information for training, validation and complete set.

\begin{tabular}{lccc}
\hline Clinical characteristic & Training set $(\mathrm{n}=70)$ & Testing set $(\mathrm{n}=70)$ & Entire set $(\mathrm{n}=140)$ \\
\hline Age (years, mean \pm SD) & $59.33 \pm 11.86$ & $60.91 \pm 14.43$ & $60.12 \pm 13.19$ \\
Sex (male/female) & $45 / 25$ & $45 / 25$ & $90 / 50$ \\
Chemotherapy (yes/no/unknown) & $20 / 43 / 7$ & $24 / 36 / 10$ & $44 / 79 / 17$ \\
Drug therapy (yes/no/unknown) & $8 / 55 / 7$ & $11 / 48 / 11$ & $19 / 103 / 18$ \\
Pharmaceutical-therapy (yes/no/unknown) & $24 / 42 / 4$ & $30 / 31 / 9$ & $54 / 73 / 13$ \\
Radiation-therapy (yes/no/unknown) & $5 / 61 / 4$ & $12 / 49 / 9$ & $17 / 110 / 13$ \\
Targeted molecular-therapy (yes/no/unknown) & $9 / 53 / 8$ & $8 / 53 / 9$ & $17 / 106 / 17$ \\
Mortality (deceased/alive) & $51 / 19$ & $51 / 19$ & $102 / 38$ \\
Overall survival time, months & $12.35 \pm 9.83$ & $13.16 \pm 11.29$ & $12.75 \pm 10.55$
\end{tabular}

SD, standard deviation.

Table II. Information on the five lncRNAs screened to build the risk assessment model.

\begin{tabular}{lcccc}
\hline lncRNA & Coefficient & HR & 95\% CI & P-value \\
\hline RP3-503A6 & -1.0585 & 2.8821 & $0.1719-0.7003$ & 0.0031 \\
LINC00940 & -1.0395 & 2.8277 & $0.1721-0.7265$ & 0.0047 \\
RP11-453M23 & 1.0178 & 0.3614 & $1.3416-5.7070$ & 0.0059 \\
AC009411 & 0.8392 & 0.4321 & $1.2657-4.2325$ & 0.0064 \\
CDRT7 & 1.0951 & 0.3345 & $1.3582-6.5803$ & 0.0065 \\
\hline
\end{tabular}

lnc, long non-coding; HR, hazard ratio.

expressed lncRNAs were screened using edgeR (Fig. 1A), whereas 167 significantly differentially expressed lncRNAs were screened using DEseq between the bad and good prognosis groups (Fig. 1B). The 117 overlapping lncRNAs were screened for further analysis (Fig. 1C).

Risk assessment model. In the training set, a total of 117 differentially expressed lncRNAs were analyzed via univariate Cox regression analysis and 35 lncRNAs were identified to be significantly correlated $(\mathrm{P}<0.05)$ with prognosis. Subsequently, multivariate Cox regression analysis was conducted for these 35 lncRNAs and 5 of them were screened to build the risk assessment model (Table II). The risk score was calculated using these five lncRNAs as follows: Risk score $=(-1.05854) \times \exp _{\mathrm{RP} 3-503 \mathrm{~A} 6}+(-1.03947) \times \exp _{\mathrm{LINC00940}}+$ $(1.017787) \times \exp _{\mathrm{RP} 11-453 \mathrm{M} 23}+(0.83919) \times \exp _{\mathrm{AC} 009411}+(1.095126)$ $\mathrm{x} \exp _{\mathrm{CDRT} 7}$

Survival analysis for five IncRNAs in training, validation and entire sets. For the training set, patients in the low-risk group were associated with longer survival time compared with the patients in the high-risk group $(15.29 \pm 11.29$ vs. $9.41 \pm 7.13$ months; $\mathrm{P}=0.0117)$. Kaplan-Meier analysis confirmed the significant difference of survival time between low-risk group and high-risk group $(\mathrm{P}<0.001$; Fig. $2 \mathrm{~A})$.

Consistent with the results of the training set, the patients in the low-risk group exhibited significantly longer survival time compared with the patients in high-risk group in the validation and the entire sets (validation set: $16.13 \pm 12.84$ vs. $10.19 \pm 8.69$ months, $\mathrm{P}=0.0273$, Fig. $2 \mathrm{~B}$; entire set: $15.71 \pm 12.01$ vs. 9.81 \pm 7.89 months, $\mathrm{P}=0.0207$, Fig. $2 \mathrm{C}$ ). Additionally, the area under the curve (AUC) was 0.910, 0.84 and 0.896 for the ROC of these 5 lncRNAs in the training set (Fig. 2D), validation set (Fig. 2E) and entire set (Fig. 2F). The Youden index was $0.600,0.621$, and 0.62 in the training, validation and entire sets, respectively.

There were significant differences in the expression levels of these five lncRNAs in training, validation and entire sets ( $\mathrm{P}<0.05$; Fig. 3). The expression levels of RP11-453M23, AC009411 and CDRT7 were significantly increased in high-risk groups, whereas the expression levels of RP3-503A6 and LINC00940 were significantly decreased compared with the low-risk group $(\mathrm{P}<0.05)$. Consistently, the results of Kaplan-Meier survival analysis demonstrated that increased expression of RP3-503A6 and LINC00940 and decreased expression of RP11-453M23, AC009411 and CDRT7 indicated improved prognoses (Fig. 4). The AUC values for ROC curves of RP3-503A6, LINC00940, RP11-453M23, AC009411 and CDRT7 were $0.691,0.679,0.615,0.652$ and 0.716 , respectively.

Prognostic factors for glioma. The results obtained from the univariate and multivariate Cox regression analysis are provided in Tables III-V. In the training, validation and entire sets, the risk score was significantly associated with the prognosis of patients and it was an independent prognostic factor. The impact of high and low risk on 

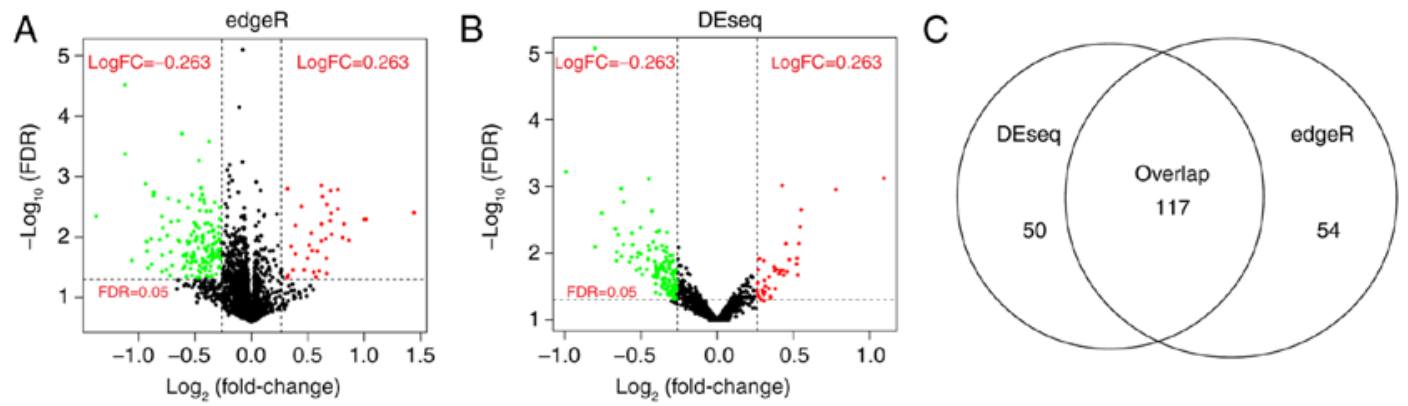

Figure 1. Significantly differentially expressed lncRNAs screened in the training set. (A) The volcanic map for lncRNAs by edgeR. (B) The volcanic map for IncRNAs by DEseq. The abscissa represents $\log _{2} \mathrm{FC}$ and the ordinate represents the negative logarithm of the P-value. The red, green and black nodes represent upregulated lncRNAs, downregulated lncRNAs and non-differentially expressed lncRNAs, respectively. (C) The overlap for lncRNAs between edgeR and DEseq. lnc, long non-coding; Fc, fold change; FDR, false discovery rate.
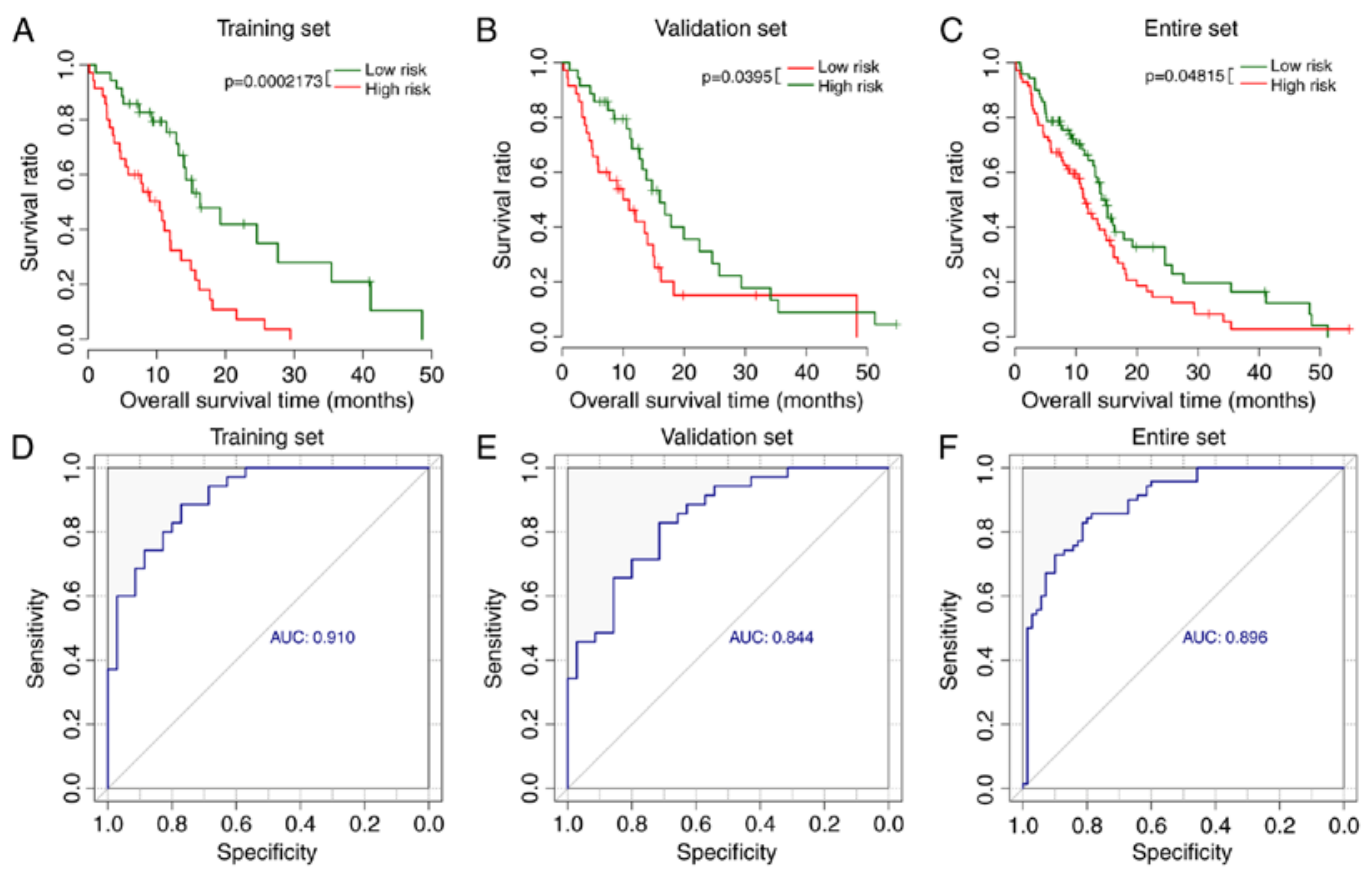

Figure 2. Kaplan-Meier curves of OS and ROC for the five lncRNAs. Kaplan-Meier curves in (A) training, (B) validation and (C) entire sets. Horizontal axis represents the survival period, and the vertical axis represents the frequency. Red lines represent the high-risk group samples, and green lines represent the low-risk group samples. ROC curves in (D) training, (E) validation and (F) entire sets. The abscissa represents sensitivity and the ordinate represents specificity. lnc, long non-coding; OS, overall survival; ROC, receiver operating characteristic; AUC, area under the curve.
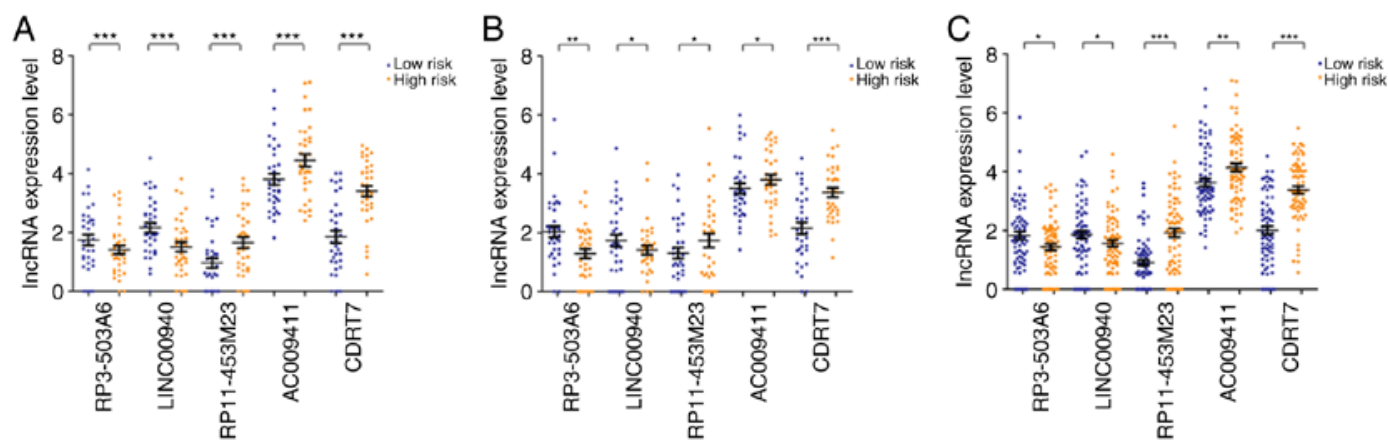

Figure 3. Expression levels of these five lncRNAs in training set, validation set and entire set. The blue dot indicates the expression values in the low risk group; the orange dot indicates the expression values in the high risk group. ${ }^{*} \mathrm{P}<0.05,{ }^{* *} \mathrm{P}<0.01$ and ${ }^{* * * *} \mathrm{P}<0.001$. lnc, long non-coding.

prognosis under the same clinical features was also analyzed using hierarchical analysis. It was demonstrated that the low-risk group had a significantly improved prognosis compared with the high-risk group in the subgroups of age $>60$ years $(P=0.00862)$, no chemotherapy $(P=0.0113)$ and no pharmaceutical therapy $(\mathrm{P}=0.0142$; Fig. 5). No 
Table III. Univariate and multivariate Cox regression analysis for malignant glioma in training set $(\mathrm{n}=70)$.

\begin{tabular}{|c|c|c|c|c|c|c|}
\hline \multirow[b]{2}{*}{ Variable } & \multicolumn{3}{|c|}{ Univariate analysis } & \multicolumn{3}{|c|}{ Multivariate analysis } \\
\hline & $\mathrm{HR}$ & $95 \% \mathrm{CI}$ & P-value & $\mathrm{HR}$ & $95 \% \mathrm{CI}$ & P-value \\
\hline \multicolumn{7}{|l|}{ Risk score } \\
\hline High/low & 1.283 & $1.116-1.474$ & 0.0002 & 1.318 & $1.108-1.568$ & 0.0019 \\
\hline \multicolumn{7}{|l|}{ Age, years } \\
\hline$\leq 60 />60$ & 1.444 & $0.823-2.534$ & 0.1978 & 2.123 & $1.088-4.142$ & 0.0273 \\
\hline \multicolumn{7}{|l|}{ Sex } \\
\hline Male/female & 0.8142 & $0.461-1.438$ & 0.4779 & 0.942 & $0.487-1.823$ & 0.8590 \\
\hline \multicolumn{7}{|l|}{ Chemotherapy } \\
\hline Yes/no & 0.3947 & $0.199-0.781$ & 0.0062 & 1.355 & $0.587-1.901$ & 0.1280 \\
\hline \multicolumn{7}{|c|}{ Pharmaceutical therapy } \\
\hline Yes/no & 0.223 & 0.0994-0.499 & 0.0003 & 0.381 & $0.261-0.446$ & 0.0092 \\
\hline
\end{tabular}

HR, hazard ratio; CI, confidence interval.

Table IV. Univariate and multivariate Cox regression analysis for malignant glioma in validation set $(n=70)$.

\begin{tabular}{|c|c|c|c|c|c|c|}
\hline \multirow[b]{2}{*}{ Variable } & \multicolumn{3}{|c|}{ Univariate analysis } & \multicolumn{3}{|c|}{ Multivariate analysis } \\
\hline & HR & $95 \% \mathrm{CI}$ & P-value & HR & $95 \% \mathrm{CI}$ & P-value \\
\hline \multicolumn{7}{|l|}{ Risk score } \\
\hline High/low & 1.186 & $0.738-1.964$ & 0.0395 & 1.147 & $0.742-1.027$ & 0.0435 \\
\hline \multicolumn{7}{|l|}{ Age, years } \\
\hline$\leq 60 />60$ & 1.772 & $0.983-3.195$ & 0.0571 & 1.264 & $0.647-2.470$ & 0.4930 \\
\hline \multicolumn{7}{|l|}{ Sex } \\
\hline Male/female & 0.862 & $0.486-1.528$ & 0.6110 & 0.939 & $0.486-1.815$ & 0.8520 \\
\hline \multicolumn{7}{|l|}{ Chemotherapy } \\
\hline Yes/no & 0.532 & $0.279-1.012$ & 0.0506 & 0.881 & $0.3312-2.339$ & 0.7990 \\
\hline \multicolumn{7}{|c|}{ Pharmaceutical therapy } \\
\hline Yes/no & 0.405 & $0.216-0.759$ & 0.0048 & 0.462 & $0.179-1.187$ & 0.1090 \\
\hline
\end{tabular}

HR, hazard ratio; CI, confidence interval.

Table V. Univariate and multivariate Cox regression analysis for malignant glioma in entire set $(n=140)$.

\begin{tabular}{|c|c|c|c|c|c|c|}
\hline \multirow[b]{2}{*}{ Variable } & \multicolumn{3}{|c|}{ Univariate analysis } & \multicolumn{3}{|c|}{ Multivariate analysis } \\
\hline & HR & $95 \% \mathrm{CI}$ & P-value & HR & $95 \% \mathrm{CI}$ & P-value \\
\hline \multicolumn{7}{|l|}{ Risk score } \\
\hline High/low & 1.037 & $0.943-1.141$ & 0.0482 & 1.447 & $0.918-2.281$ & 0.0197 \\
\hline \multicolumn{7}{|l|}{ Age, years } \\
\hline$\leq 60 />60$ & 1.57 & $1.052-2.344$ & 0.0272 & 1.447 & $0.918-2.281$ & 0.1120 \\
\hline \multicolumn{7}{|l|}{ Sex } \\
\hline Male/female & 0.815 & $0.547-1.215$ & 0.3160 & 0.841 & $0.539-1.312$ & 0.4460 \\
\hline \multicolumn{7}{|l|}{ Chemotherapy } \\
\hline Yes/no & 0.485 & $0.308-0.762$ & 0.0017 & 1.414 & $0.641-3.119$ & 0.3910 \\
\hline \multicolumn{7}{|c|}{ Pharmaceutical therapy } \\
\hline Yes/no & 0.339 & $0.214-0.539$ & $<0.0001$ & 0.253 & $0.114-0.563$ & 0.0008 \\
\hline
\end{tabular}

HR, hazard ratio; CI, confidence interval. 

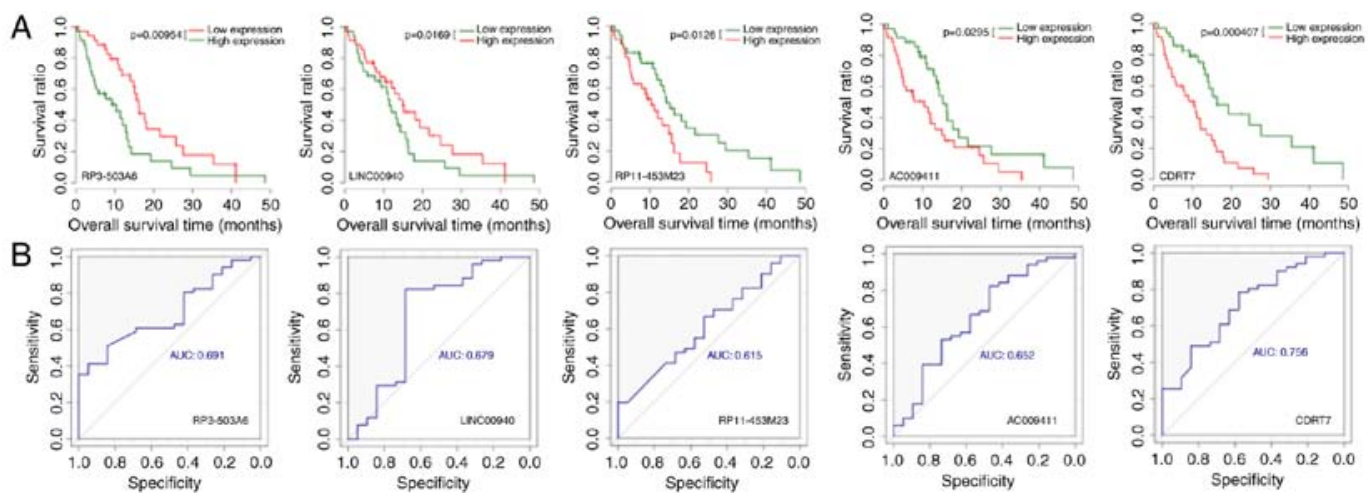

Figure 4. (A) Kaplan-Meier overall survival curves. (B) ROC curves for RP3-503A6, LINC00940, RP11-453M23, AC009411 and CDRT7. For Kaplan-Meier curves, the horizontal axis represents the survival period and the vertical axis represents the frequency. Red lines represent the high-risk group samples, green lines represent the low-risk group samples. For ROC curves, the abscissa represents sensitivity and the ordinate represents specificity. ROC, receiver operating characteristic; AUC, area under the curve.
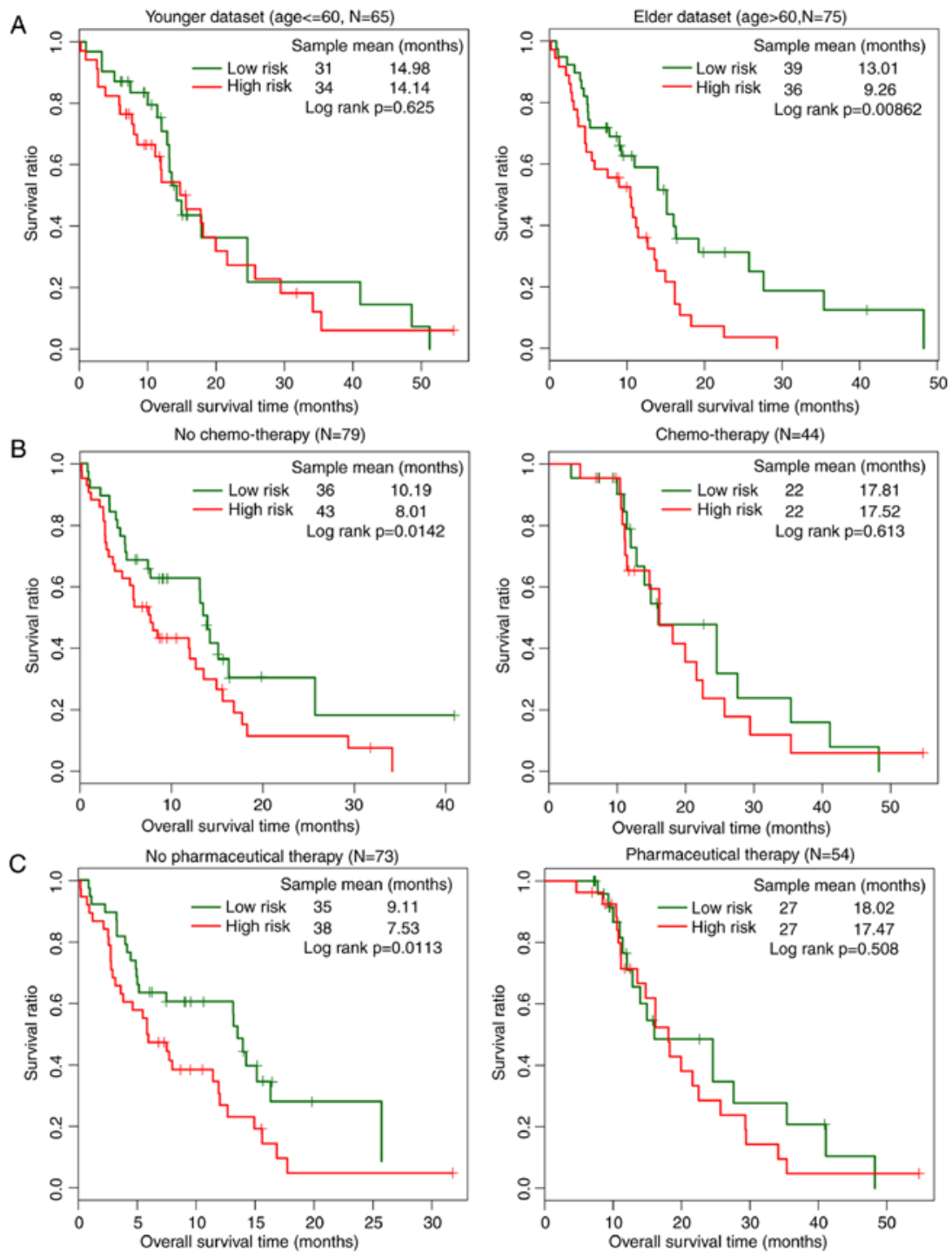

Figure 5. Kaplan-Meier curves of overall survival between high-risk and low-risk patients in the entire dataset based on the hierarchical analysis. (A) Kaplan-Meier curves for samples $<60$ years old (left) and age over 60 years old (right). (B) Kaplan-Meier curves for samples without chemotherapy (left) and with chemotherapy (right). (C) Kaplan-Meier curves for samples without pharmaceutical therapy (left) and with pharmaceutical therapy. The horizontal axis represents the survival period; the vertical axis represents the frequency. Red lines represent the high-risk group samples; green lines represent the low-risk group samples.

significant differences of survival ratio were observed between low-risk and high-risk groups in the subgroups of age $<60$ years $(\mathrm{P}=0.625)$, chemo-therapy $(\mathrm{P}=0.613)$ and pharmaceutical therapy ( $\mathrm{P}=0.508$; Fig. 5). 
A
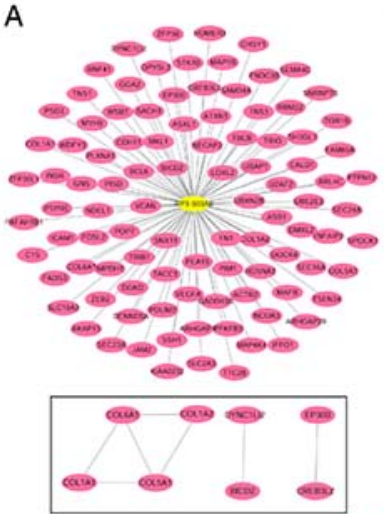

D

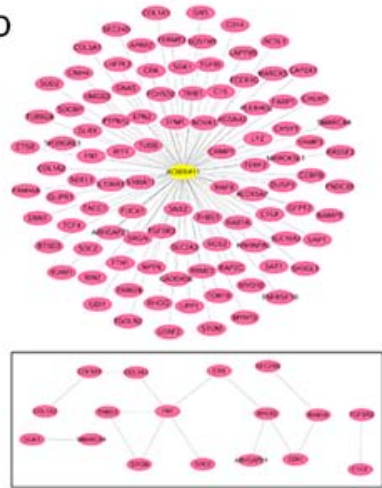

B

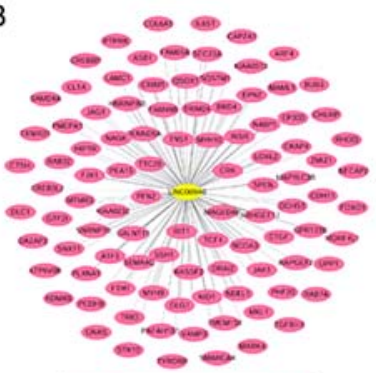

C

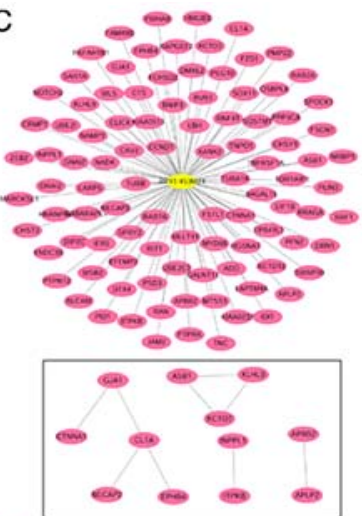

E
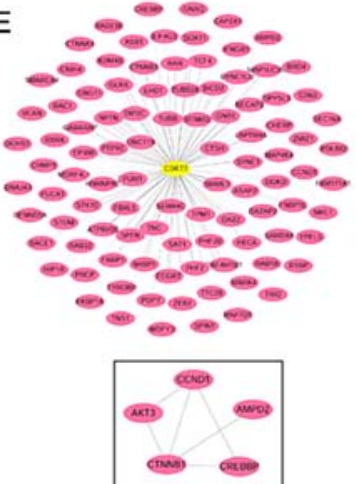

Figure 6. Co-expression networks for (A) RP3-503A6, (B) LINC00940, (C) RP11-453M23, (D) AC009411 and (E) CDRT7. The bottom of each network diagram provides the mRNA interaction in the co-expression network.

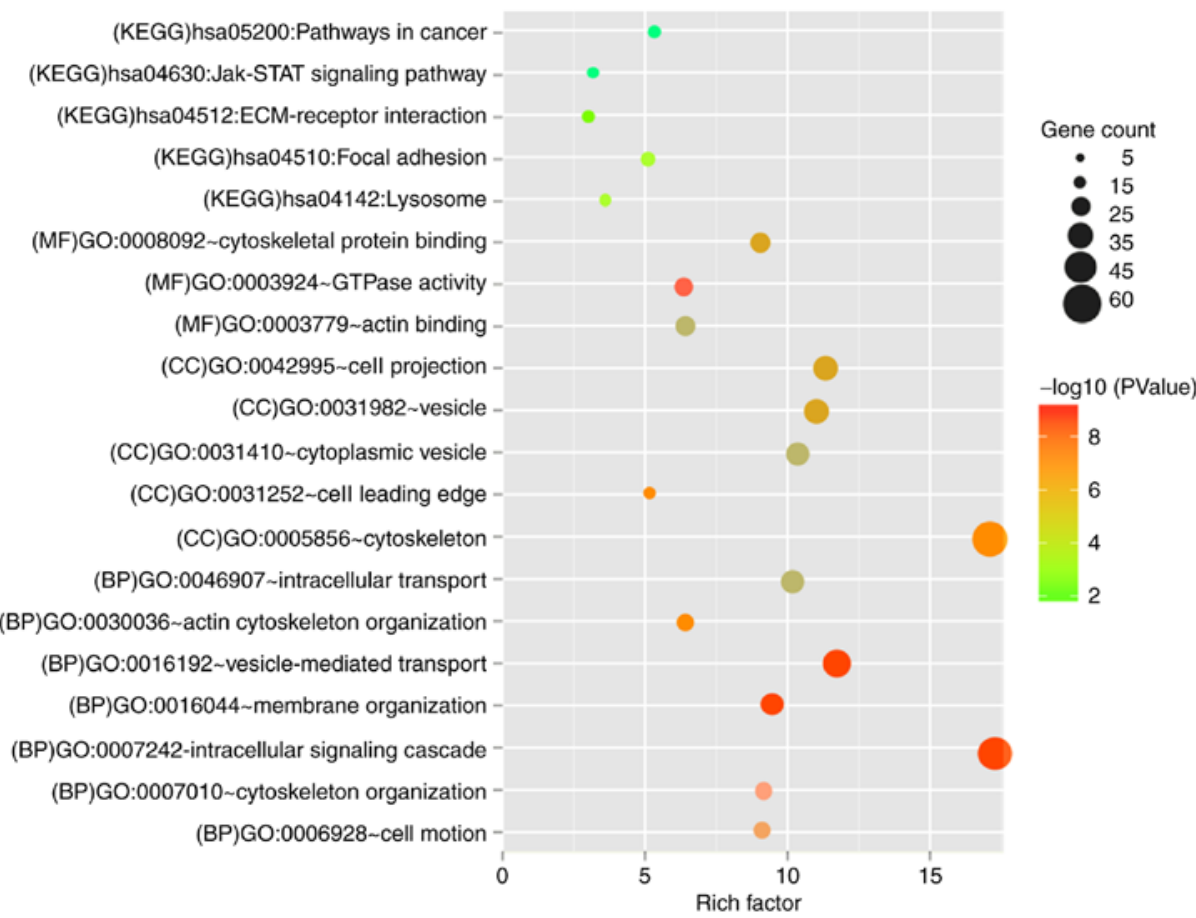

Figure 7. Gene Ontology and Kyoto Encyclopedia of Genes and Genomes term analysis of potential genes associated with five lncRNAs. The rich factor demonstrates the degree of enrichment, which was calculated by the following formula: (The number of selected genes in a term/total number of selected genes)/(the total number of genes in a term of the database/the total number of genes in the database). The Node size represents the number of selected genes and color represents the P-value of the enrichment analysis. CC, cellular component; MF, molecular function; BP, biological process; lnc, long non-coding.

Functional enrichment for co-expressed mRNAs of signature lncRNAs. The co-expressed network for each lncRNA is demonstrated in Fig. 6. GO functional biology processes and KEGG pathways enriched by genes in the networks are shown in Fig. 7. The genes were involved in membrane organization, vesicle-mediated transport, intracellular signaling 
cascade, cytoskeleton organization and cell motion biological process (BP) terms. For cell component (CC) term, cell leading edge, cytoskeleton, cell projection, vesicle and cytoplasmic vesicle were the significantly enriched ones. For the molecule function (MF) term, GTPase activity, cytoskeletal protein binding and actin binding were important. It was also noted that the genes associated with these five lncRNAs were mainly enriched in cell adhesion (hsa04510: Focal adhesion) and Janus kinase (Jak)-signal transducer and activator of transcription (STAT) signaling pathway (hsa04630: Jak-STAT signaling pathway).

\section{Discussion}

A total of 117 lncRNAs were identified between bad and good prognosis groups in the training set using edgeR and DEseq packages. According to univariate and multivariate Cox regression analyses, five lncRNAs were screened to establish a risk assessment model. The risk assessment model demonstrated good prognostic function with increased AUC in training, validation and entire sets. There were significant differences in the expression levels of these five lncRNAs in the training, validation and entire set. Furthermore, risk score calculated using these five lncRNAs was certified as an independent prognostic factor for glioma. According to the results of hierarchical analysis, the prognostic function of the risk assessment model may be useful for patients $>60$ years who did not receive chemotherapy and pharmaceutical therapy.

Multiple genes were screened to be co-expressed with these five lncRNAs and these were mainly involved in cell adhesion, Jak-STAT signaling pathway, intracellular signaling cascade, cell motion, cytoskeleton, GTPase activity, cytoskeletal protein binding and actin binding.

Metastasis accounts for the vast majority of cancer-associated mortalities and is a result of cancer cells moving from the primary site (site of the origin of cancer) to a distant site or other organ (25). The spatial and temporal reorganization of the cytoskeleton serves a critical role in the movements and alterations of cell shape throughout the complex processes of metastasis (26). The importance of cytoskeleton-associated proteins and pathways involved in glioma has also been reported in numerous previous studies $(27,28)$. In addition, increasing evidence revealed that IncRNAs are associated with the occurrence and development of glioma by regulating the structure of reorganization of cytoskeleton-associated proteins and signaling pathways (29,30). Additionally, the association between metastasis or cytoskeleton and the prognosis of glioma has also been reported $(31,32)$. According to our analysis, the biological process of cytoskeleton organization and actin cytoskeleton organization, the cell component of cytoskeleton, and the molecular function of cytoskeletal protein binding were enriched by the genes associated with the five IncRNAs of the constructed risk assessment model. Therefore, it may be reasonable to infer that the cytoskeleton-associated process may be one of the underlying mechanisms for the prognostic risk assessment model in glioma.

Consistently, the focal adhesion pathway, which is associated with cell invasion and metastasis, was screened as a significant pathway enriched by the genes associated with the five lncRNAs based on the KEGG analysis. Focal adhesions are known as the large macromolecular assemblies through which regulatory signals and mechanical forces are transmitted between interacting cells and the extracellular matrix (ECM) (33). Focal adhesion functions as the mechanical linkage to the ECM and as a biochemical signaling hub to concentrate and direct numerous signaling proteins at sites of integrin binding and clustering (34). Yue et al (35) demonstrated that a cytoskeleton protein of microtubules ending-binding 2 (EB2) may regulate the dynamics of focal adhesion through $M A P 4 K 4$. Importantly, adhesion to ECM has been demonstrated to contribute to the resistance to chemotherapy and radiotherapy in tumor cells $(36,37)$. Therefore, focal adhesion and ECM may have a prognostic role for tumors (38).

The Jak-STAT signaling pathway is associated with multiple biological processes, including cell survival, growth, differentiation and development (39). In the central nervous system, the Jak-STAT signaling pathway is mainly associated with gene regulation during development, inflammation, hormone release and tumorigenesis (39). A previous study reported that inhibiting the STAT3 signaling pathway resulted in depressed proliferation and increased apoptosis in various cancer types including glioma (40). It was speculated that the regulation of the Jak-STAT signaling pathway may be an important factor for the prognostic function of the risk assessment model in glioma.

Multiple genes, including metastasis suppressor protein 1 (MTSS1), drebrin 1 (DBN1), transforming growth factor $\beta 1$ induced transcript 1 (TGFB1I1), and cyclin D1 (CCND1) are associated with cytoskeleton and adhesion or Jak-STAT signaling pathway-associated processes. MTSS1 is reported to be associated with the progression and metastasis of tumor in multiple organ sites, possibly via an interaction with the actin cytoskeleton (41). DBN1 serves a role in cell migration, the extension of neuronal processes and plasticity of dendrites and is overexpressed during cancer metastasis (42). TGFB1II functions as a molecular adapter for coordinating various protein-protein interactions associated with focal adhesion (43). Multidimensional analysis of gene expression reveals that TGFB1I1-induced EMT contributes to the malignant progression of astrocytomas (44). The activation of CCND1 affects the prognosis of glioma by promoting cell proliferation, migration and invasion (45). Therefore, it was inferred that the lncRNAs in the risk assessment model may serve critical functions for the prognosis of glioma by regulating the expression or function of these genes. The prognosis of glioma patients may be predicted by detecting the expressions of the five lncRNAs.

To conclude, lncRNAs serve important roles in the development and progression of tumors and may affect tumor prognosis. The present study provided a risk assessment model consisting of five differently expressed lncRNAs, which may help to assess the prognosis of glioma with increased accuracy, as suggested by the survival analysis and increased AUC value. The risk assessment model indicates the cytoskeleton, adhesion and Jak-STAT signaling pathway may be the most important mechanism in glioma prognosis. However, as all expression data were downloaded from only one database, there may be possible bias in the present study and further studies are required to verify the conclusions. 


\section{Acknowledgements}

Not applicable.

\section{Funding}

No funding was received.

\section{Availability of data and materials}

The datasets used and/or analyzed during the present study are available from the corresponding author on reasonable request.

\section{Authors' contributions}

$\mathrm{CH}$ performed data analyses and wrote the manuscript. $\mathrm{YZ}$ and CL collected the dataset, contributed to data analyses and manuscript revision. YK conceived and designed the study. All authors read and approved the final manuscript.

\section{Ethics approval and consent to participate}

In the original creation of the datasets, the local institutional review boards of all participating centers approved the trials and informed consent to participate was obtained from all patients.

\section{Patient consent for publication}

Not applicable.

\section{Competing interests}

The authors declare that they have no competing interests.

\section{References}

1. Sampetrean $\mathrm{O}$ and Saya $\mathrm{H}$ : Modeling phenotypes of malignant gliomas. Cancer Sci 109: 6-14, 2017.

2. Louis DN: Molecular pathology of malignant gliomas. Ann Rev Pathol 1: 97-117, 2006.

3. Wang P, Ren Z and Sun P: Overexpression of the long non-coding RNA MEG3 impairs in vitro glioma cell proliferation. J Cell Biochem 113: 1868-1874, 2012.

4. Zhao W, Song M, Zhang J, Kuerban M and Wang H: Combined identification of long non-coding RNA CCAT1 and HOTAIR in serum as an effective screening for colorectal carcinoma. Int J Clin Exp Pathol 8: 14131-14140, 2015.

5. Zhou J, Li X, Wu M, Lin C, Guo Y and Tian B: Knockdown of long noncoding RNA GHET1 inhibits cell proliferation and invasion of colorectal cancer. Oncol Res 23: 303-309, 2016.

6. Gibb EA, Brown CJ and Lam WL: The functional role of long non-coding RNA in human carcinomas. Mol Cancer 10: 38, 2011.

7. Martens-Uzunova ES, Böttcher R, Croce CM, Jenster G, Visakorpi T and Calin GA: Long noncoding RNA in prostate, bladder, and kidney cancer. Eur Urol 65: 1140-1151, 2014.

8. Li F, Cao L, Hang D, Wang F and Wang Q: Long non-coding RNA HOTTIP is up-regulated and associated with poor prognosis in patients with osteosarcoma. Int J Clin Exp Pathol 8: 11414-11420, 2015.

9. Jariwala N and Sarkar D: Emerging role of lncRNA in cancer: A potential avenue in molecular medicine. Ann Transl Med 4: 286, 2016.

10. Wan L, Zhang L, Fan K, Cheng ZX, Sun QC and Wang JJ: Knockdown of long noncoding RNA PCAT6 inhibits proliferation and invasion in lung cancer cells. Oncol Res 24: 161-170, 2016.
11. Chen S, Wu H, Lv N, Wang H, Wang Y, Tang Q, Shao H and Sun C: LncRNA CCAT2 predicts poor prognosis and regulates growth and metastasis in small cell lung cancer. Biomed Pharmacother 82: 583-588, 2016.

12. Bian EB, Li J, Xie YS, Zong G, Li J and Zhao B: LncRNAs: New players in gliomas, with special emphasis on the interaction of lncRNAs With EZH2. J Cell Physiol 230: 496-503, 2015.

13. Yao J, Zhou B, Zhang J, Geng P, Liu K, Zhu Y and Zhu W: A new tumor suppressor LncRNA ADAMTS9-AS2 is regulated by DNMT1 and inhibits migration of glioma cells. Tumour Biol 35: 7935-7944, 2014.

14. Zhang XQ and Leung GK: Long non-coding RNAs in glioma: Functional roles and clinical perspectives. Neurochem Int 77: 78-85, 2014.

15. Ma KX, Wang HJ, Li XR, Li T, Su G, Yang P and Wu JW: Long noncoding RNA MALAT1 associates with the malignant status and poor prognosis in glioma. Tumour Biol 36: 3355-3359, 2015.

16. Hu L, Lv QL, Chen SH, Sun B, Qu Q, Cheng L, Guo Y, Zhou HH and Fan L: Up-regulation of long non-coding RNA AB073614 predicts a poor prognosis in patients with glioma. Int J Environ Res Public Health 13: 433, 2016.

17. Harrow J, Frankish A, Gonzalez JM, Tapanari E, Diekhans M, Kokocinski F, Aken BL, Barrell D, Zadissa A, Searle S, et al: GENCODE: The reference human genome annotation for The ENCODE Project. Genome Res 22: 1760-1774, 2012.

18. Anders $\mathrm{S}$ and Huber W: Differential expression analysis for sequence count data. Genome Biol 11: R106, 2010.

19. Robinson MD, McCarthy DJ and Smyth GK: edgeR: A Bioconductor package for differential expression analysis of digital gene expression data. Bioinformatics 26: 139-140, 2010.

20. Adler P, Kolde R, Kull M, Tkachenko A, Peterson H, Reimand J and Vilo J: Mining for coexpression across hundreds of datasets using novel rank aggregation and visualization methods. Genome Biol 10: R139, 2009.

21. Kolde R, Laur S, Adler P and Vilo J: Robust rank aggregation for gene list integration and meta-analysis. Bioinformatics 28 : 573-580, 2012.

22. Franceschini A, Szklarczyk D, Frankild S, Kuhn M, Simonovic M, Roth A, Lin J, Minguez P, Bork P, von Mering C, et al: STRING v9.1: Protein-protein interaction networks, with increased coverage and integration. Nucleic Acids Res 41: D808-D815, 2013.

23. Su G, Morris JH, Demchak B and Bader GD: Biological network exploration with Cytoscape 3. Curr Protoc Bioinformatics 47: 8.13.11-24, 2014.

24. Huang da W, Sherman BT and Lempicki RA: Systematic and integrative analysis of large gene lists using DAVID bioinformatics resources. Nat Prot 4: 44-57, 2009.

25. Schroeder A, Heller DA, Winslow MM, Dahlman JE, Pratt GW, Langer R, Jacks T and Anderson DG: Treating metastatic cancer with nanotechnology. Nat Rev Cancer 12: 39-50, 2011.

26. Fife CM, McCarroll JA and Kavallaris M: Movers and shakers: Cell cytoskeleton in cancer metastasis. Br J Pharmacol 171: 5507-5523, 2014.

27. Fujimura A, Michiue H, Cheng Y, Uneda A, Tani Y, Nishiki T, Ichikawa T, Wei FY, Tomizawa K and Matsui H: Cyclin G2 promotes hypoxia-driven local invasion of glioblastoma by orchestrating cytoskeletal dynamics. Neoplasia 15: 1272-1281, 2013.

28. Zheng PP, Sieuwerts AM, Luider TM, van der Weiden M, Sillevis-Smitt PA and Kros JM: Differential expression of splicing variants of the human caldesmon gene $(C A L D 1)$ in glioma neovascularization versus normal brain microvasculature. Am J Pathol 164: 2217-2228, 2004.

29. Cardama GA, Gonzalez N, Ciarlantini M, Gandolfi Donadío L, Comin MJ, Alonso DF, Menna PL and Gomez DE: Proapoptotic and antiinvasive activity of Rac1 small molecule inhibitors on malignant glioma cells. Onco Targets Ther 7: 2021-2033, 2014.

30. Han J, Liu S, Sun Z, Zhang Y, Zhang F, Zhang C, Shang D, Yang H, Su F, Xu Y, et al: LncRNAs2Pathways: Identifying the pathways influenced by a set of lncRNAs of interest based on a global network propagation method. Sci Rep 7: 46566, 2017.

31. Han MZ, Huang B, Chen AJ, Zhang X, Xu R, Wang J and Li XG: High expression of RAB43 predicts poor prognosis and is associated with epithelial-mesenchymal transition in gliomas. Oncol Rep 37: 903-912, 2017. 
32. Shan S, Hui G, Hou F, Shi H, Zhou G, Yan H, Wang L and Liu J: Expression of metastasis-associated protein 3 in human brain glioma related to tumor prognosis. Neurol Sci 36: 1799-1804, 2015.

33. Chen CS, Alonso JL, Ostuni E, Whitesides GM and Ingber DE: Cell shape provides global control of focal adhesion assembly. Biochem Biophys Res Commun 307: 355-361, 2003.

34. Wehrle-Haller B: Structure and function of focal adhesions. Curr Opin Cell Biol 24: 116-124, 2012.

35. Yue J, Xie M, Gou X, Lee P, Schneider MD and Wu X: Microtubules regulate focal adhesion dynamics through MAP4K4. Dev Cell 31: 572-585, 2014.

36. Park CC, Zhang H, Pallavicini M, Gray JW, Baehner F, Park CJ and Bissell MJ: Beta1 integrin inhibitory antibody induces apoptosis of breast cancer cells, inhibits growth, and distinguishes malignant from normal phenotype in three dimensional cultures and in vivo. Cancer Res 66: 1526-1535, 2006.

37. Eke I, Deuse Y, Hehlgans S, Gurtner K, Krause M, Baumann M, Shevchenko A, Sandfort V and Cordes N: $\beta_{1}$ Integrin/FAK/cortactin signaling is essential for human head and neck cancer resistance to radiotherapy. J Clin Invest 122: 1529-1540, 2012.

38. Shimizu T, Kurozumi K, Ishida J, Ichikawa T and Date I: Adhesion molecules and the extracellular matrix as drug targets for glioma. Brain Tumor Pathol 33: 97-106, 2016.

39. Nicolas CS, Amici M, Bortolotto ZA, Doherty A, Csaba Z, Fafouri A, Dournaud P, Gressens P, Collingridge GL, et al: The role of JAK-STAT signaling within the CNS. JAKSTAT 2: e22925, 2013.

40. Gu J, Li G, Sun T, Su Y, Zhang X, Shen J, Tian Z and Zhang J: Blockage of the STAT3 signaling pathway with a decoy oligonucleotide suppresses growth of human malignant glioma cells. J Neurooncol 89: 9-17, 2008.
41. Woodings JA, Sharp SJ and Machesky LM: MIM-B, a putative metastasis suppressor protein, binds to actin and to protein tyrosine phosphatase delta. Biochem J 371: 463-471, 2003.

42. Lin Q, Tan HT, Lim TK, Khoo A, Lim KH and Chung MC: iTRAQ analysis of colorectal cancer cell lines suggests Drebrin (DBN1) is overexpressed during liver metastasis. Proteomics 14: 1434-1443, 2014

43. Wang X, Hu G, Betts C, Harmon EY, Keller RS, Van De Water L and Zhou J: Transforming growth factor- $\beta 1$-induced transcript 1 protein, a novel marker for smooth muscle contractile phenotype, is regulated by serum response factor/myocardin protein. $\mathrm{J}$ Biol Chem 286: 41589-41599, 2011.

44. Liu Y, Hu H, Wang K, Zhang C, Wang Y, Yao K, Yang P, Han L, Kang C, Zhang W and Jiang T: Multidimensional analysis of gene expression reveals TGFB1I1-induced EMT contributes to malignant progression of astrocytomas. Oncotarget 5: 12593-12606, 2014.

45. Alqudah MA, Agarwal S, Al-Keilani MS, Sibenaller ZA, Ryken TC and Assem M: NOTCH3 is a prognostic factor that promotes glioma cell proliferation, migration and invasion via activation of CCND1 and EGFR. PLoS One 8: e77299, 2013.

This work is licensed under a Creative Commons Attribution-NonCommercial-NoDerivatives 4.0 International (CC BY-NC-ND 4.0) License. 